\title{
ShelXIe: a Qt graphical user interface for SHELXL
}

${ }^{a}$ Christian B. Hübschle

aLaboratory of Crystallography, University of Bayreuth, Bayreuth, Germany chuebsch@moliso.de

ShelXle [1] is a graphical user interface for SHELXL [2], currently the most widely used program for small-molecule structure refinement. It combines an editor with syntax highlighting for the $S H E L X L$-associated ins (input) and res (output) files with an interactive graphical display for visualization of a three-dimensional structure including the electron density $\left(F_{0}\right)$ and difference density $\left(F_{0}-F_{\mathrm{c}}\right)$ maps. Special features of ShelXle include intuitive atom (re-)naming, structure visualization and a novel way of displaying disorder extending over special positions. The DSR [3, 4] plugin can be of great help in mastering messy disorders. ShelXIe is completely compatible with all features of SHELXL and is written entirely in C++ using the Qt4 and FFTW libraries. It is available at no cost for Windows, Linux and Mac-OS $X$ and as source code. Since its release in 2011 it has been 47900 times downloaded.

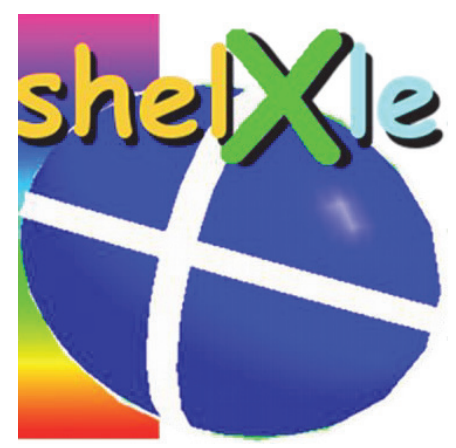

Fig. 1. ShelXle's Icon

\section{References:}

[1]C. B. Hübschle, G. M. Sheldrick and B. Dittrich, (2011) J. Appl. Cryst., 44, 1281-1284.

[2]G. M. Sheldrick, (2008). Acta Cryst. A64, 112-122.

[3]D. Kratzert, I. Krossing, (2018) J. Appl. Cryst. , 51, 928-934.

[4]D. Kratzert, J.J. Holstein, I. Krossing, (2015) J. Appl. Cryst. 48, 933-938. 